\title{
Stationary Solutions of Incompressible Stokes Equation for a Circular Pipe
}

M. Heil

Fachbereich Physik der Philipps-Universität Marburg

Z. Naturforsch. 42 a, 543-546 (1987); received January 23, 1987

Stationary modes of the incompressible Stokes equation are derived using the method of potentials. Their relation to instationary modes is discussed.

In a recent paper [1] a method developed by Hansen, Stratton, Morse, Feshbach [2] ("HSMFmethod") for solving linear vector-wave equations in electrodynamics was applied to find analytical expressions for three-dimensional time-dependent solutions of the Stokes equation for a pipe with circular cross-section. In this paper we derive analytical expressions for stationary modes of the stationary Stokes equation

$$
\frac{1}{\varrho_{0}} \frac{\partial p}{\partial \boldsymbol{r}}=-v_{0} \operatorname{rot} \operatorname{rot} \boldsymbol{u}, \operatorname{div} \boldsymbol{u}=0
$$

with the help of "potentials" $a, b$ for the same geometry and boundary condition as in [1].

Expressing $\boldsymbol{u}$ and $p$ by potentials $a(\boldsymbol{r}, t), b(\boldsymbol{r}, t)$ ([1], ref. 24)

$\boldsymbol{u}=\operatorname{rot} \boldsymbol{a}+\operatorname{rot} \operatorname{rot} \boldsymbol{b}, \quad p=p_{0}+\varrho_{0} v_{0} \operatorname{div}\left(\boldsymbol{e}_{z} \Delta b\right)$,

$\boldsymbol{a}=a \boldsymbol{e}_{z}, \quad \boldsymbol{b}=b \boldsymbol{e}_{z}$,

we get for $a, b$ the differential equations

$$
\Delta a=0, \Delta \Delta b=0 .
$$

Introducing cylindrical coordinates $r, \varphi, z$ and separating $a(r, \varphi, z)$ and $b(r, \varphi, z)$ into $a(r, \varphi, z)=$ $a_{r}(r) a_{\varphi}(\varphi) a_{z}(z), \quad b(r, \varphi, z)=b_{r}(r) b_{\varphi}(\varphi) b_{z}(z) \quad$ we find for $k \neq 0$ the solutions

$$
\begin{aligned}
a(r, \varphi, z)= & a_{m} J_{m}(i k r) \mathrm{e}^{i m \varphi} \mathrm{e}^{i k z}, \\
b(r, \varphi, z)= & b_{m} J_{m}(i k r) \mathrm{e}^{i m \varphi} \mathrm{e}^{i k z} \\
& +\frac{c_{m} r}{i k} J_{m}^{\prime}(i k r) \mathrm{e}^{i m \varphi} \mathrm{e}^{i k z},
\end{aligned}
$$

Reprint requests to Dr. Heil, Fachbereich Physik der Philipps-Universität, Renthof 6, 3550 Marburg. which give

$$
\begin{aligned}
u_{r}= & {\left[a_{m} \frac{i m}{r} J_{m}+b_{m} k^{2}\left(J_{m+1}-\frac{m}{i k r} J_{m}\right)\right.} \\
& \left.+c_{m}\left(\frac{m^{2}}{i k r}-i k r\right) J_{m}\right] \mathrm{e}^{i m \varphi} \mathrm{e}^{i k z}, \\
u_{\varphi}= & {\left[a_{m} i k\left(J_{m+1}-\frac{m}{i k r} J_{m}\right)-b_{m} \frac{m k}{r} J_{m}\right.} \\
& \left.+c_{m} i m\left(\frac{m}{i k r} J_{m}-J_{m+1}\right)\right] \mathrm{e}^{i m \varphi} \mathrm{e}^{i k z}, \\
u_{z}= & -b_{m} k^{2} J_{m} \\
& \left.+c_{m}\left\{(2+m) J_{m}-i k r J_{m+1}\right\}\right] \mathrm{e}^{i m \varphi} \mathrm{e}^{i k z} .
\end{aligned}
$$

The boundary condition $\boldsymbol{u}(r=R, \varphi, z)=\mathbf{0}$ leads to the dispersion relation

$$
\begin{aligned}
D_{\mathrm{S}}(k, m):= & i k R m J_{m}^{3}(i k R) \\
& +\left[k^{2} R^{2}-2 m(2+m)\right] J_{m}^{2} J_{m+1} \\
& +i k R(2+3 m) J_{m} J_{m+1}^{2} \\
& +k^{2} R^{2} J_{m+1}^{3}=0, \\
m & =0,1,2, \ldots
\end{aligned}
$$

For $m=0$ we find

$$
J_{1}\left[J_{0}^{2}+\frac{2 i}{k R} J_{0} J_{1}+J_{1}^{2}\right]=0,
$$

which means either

a)

$$
\begin{array}{ll}
\text { a) } & J_{1}\left(i k^{(j)} R\right)=0, \quad k^{(j)}=i k_{i}^{(j)}, \\
& \left(k_{i}^{(j)}>0 \text { for } z>0\right), \\
& a_{0} \neq 0, \quad b_{0}=c_{0}=0, \quad j=1,2,3, \ldots \\
\text { or } & \\
\text { b) } & J_{0}^{2}+\frac{2 i}{k^{(j)} R} J_{0} J_{1}+J_{1}^{2}=0 \Leftrightarrow J_{0} J_{2}=J_{1}^{2}, \\
& a_{0}=0, \quad b_{0} \neq 0, \quad c_{0} \neq 0 .
\end{array}
$$

See also [3], p. 547. 
Solutions $\boldsymbol{u}(r, \varphi, z)$ which belong to a) or b) are given by

a)

$$
\begin{aligned}
\boldsymbol{u}^{(0, j)} & =-\boldsymbol{e}_{\varphi} a_{0} i k^{(j)} J_{1}\left(i k^{(j)} r\right) \mathrm{e}^{i k^{(j)} z} \\
& =-\boldsymbol{e}_{\varphi} \tilde{a}_{0}^{(j)} J_{1}\left(k_{i}^{(j)} r\right) \mathrm{e}^{-k_{i}^{(j)} z}, \\
\tilde{a}_{0}^{(j)} & =a_{0} k_{i}^{(j)},
\end{aligned}
$$

b) $\quad u_{r}^{(0, j)}=\tilde{b}_{0}^{(j)}\left[J_{1}\left(i k^{(j)} r\right)\right.$

$$
\left.-\frac{r}{R} \frac{J_{0}\left(i k^{(j)} r\right)}{J_{0}\left(i k^{(j)} R\right)} J_{1}\left(i k^{(j)} R\right)\right] \mathrm{e}^{i k^{(j)} z},
$$$$
u_{\varphi}^{(0, j)}=0,
$$

$$
\begin{aligned}
& u_{z}^{(0, j)}=\tilde{b}_{0}^{(j)}\left[\frac{\left\{2 J_{0}\left(i k^{(j)} r\right)-i k^{(j)} r J_{1}\left(i k^{(j)} r\right)\right\} J_{0}\left(i k^{(j)} R\right)}{2 J_{0}\left(i k^{(j)} R\right)-i k^{(j)} R J_{1}\left(i k^{(j)} R\right)}-J_{0}\left(i k^{(j)} r\right)\right] \mathrm{e}^{i k^{(j)} z}, \\
& a_{0}=0, \quad c_{0}=\frac{b_{0}\left(k^{j}\right)^{2} J_{1}\left(i k^{(j)} R\right)}{i k^{(j)} R J_{0}\left(i k^{(j)} R\right)} .
\end{aligned}
$$

Solutions for $m \neq 0$ can be found from (5) with

$$
\begin{aligned}
& c_{m}^{(j)}=\tilde{b}_{m}^{(j)} \frac{J_{m}\left(i k^{(j)} R\right)}{(2+m) J_{m}\left(i k^{(j)} R\right)-i k^{(j)} R J_{m+1}\left(i k^{(j)} R\right)}, \\
& a_{m}^{(j)}=\tilde{b}_{m}^{(j)} \frac{i R}{m J_{m}\left(i k^{(j)} R\right)} \frac{\left(J_{m+1}-\frac{m}{i k^{(j)} R} J_{m}\right)\left[(2+m) J_{m}-i k^{(j)} R J_{m+1}\right]+\left(\frac{m^{2}}{i k^{(j)} R}-i k^{(j)} R\right) J_{m}^{2}}{(2+m) J_{m}\left(i k^{(j)} R\right)-i k^{(j)} R J_{m+1}\left(i k^{(j)} R\right)},
\end{aligned}
$$

where $k^{(j)}$ are solutions of $(6)$ and $\tilde{b}_{m}^{(j)}=b_{m}\left(k^{(j)}\right)^{2}$. The stationary pressure functions $p^{(m, j)}(r, \varphi, z)$ for $k^{(j)} \neq 0$, which follow from (2), (4) are given by

$$
\begin{aligned}
p^{(m, j)}(r, \varphi, z)= & p_{0}-2 \varrho_{0} v_{0} i k^{(j)} c_{m}^{(j)} \\
& \cdot J_{m}\left(i k^{(j)} r\right) \mathrm{e}^{i m \varphi} \mathrm{e}^{i k^{(j)} z} .
\end{aligned}
$$

If $k^{(j)}$ is a solution of (6) the same holds for $-k^{(j)}$. Numerical calculations for $m=0$ ([1]) with small but finite values of $\sigma$ are in agreement with (7a) to $(8 \mathrm{~b})$. As can be seen from (6), there exist no solutions for $k^{(j)}$ with

$$
\begin{aligned}
J_{m}^{\prime}\left(i k^{(j)} R\right)= & -J_{m+1}\left(i k^{(j)} R\right) \\
& +\frac{m}{i k^{(j)} R} J_{m}\left(i k^{(j)} R\right)=0, m>0,
\end{aligned}
$$

as might be supposed from Figs. 1, 2 in [1].

The analytical limit process $\sigma \rightarrow 0$ applied to (19) of [1], where $\sigma$ is the time-separation constant, does not give the dispersion relation for the stationary

$$
\begin{aligned}
\varphi, z, t, k, \sigma)=\mathbf{0} & \\
\boldsymbol{u}^{(m, j)}(r, \varphi, z) & =\left.\frac{\partial\left[\boldsymbol{u}_{(r, \varphi, z, t, k, \sigma)}^{(m)} \mathrm{e}^{\sigma t}\right]}{\partial \sigma}\right|_{\substack{\sigma=0 \\
k=k^{(j)}}} \cdot A\left(m, k^{(j)}\right) \quad\left(a_{0}=0 \text { for } m=0\right) .
\end{aligned}
$$

$A\left(m, k^{(j)}\right)$ are constants and $k^{(j)}$ is a solution of (6). The only exception of relation (12) is the stationary solution for $m=0, a_{0} \neq 0, b_{0}=c_{0}=0$. In this special case $\boldsymbol{u}^{(0, j)}(r, z)$ follows directly from

$$
\boldsymbol{u}^{(0)}(r, z, t, k, \sigma)
$$

$=-\boldsymbol{e}_{\varphi} a_{0} \sqrt{\frac{\sigma}{v_{0}}-k^{2}} J_{0}^{\prime}\left(\sqrt{\frac{\sigma}{v_{0}}-k^{2}}\right) \mathrm{e}^{i k z} \mathrm{e}^{-\sigma t}$

for $\sigma=0$ since $\boldsymbol{u}^{(0)}(r, z, t, k, \sigma=0) \neq \mathbf{0}$. For $\sigma=0$ ( $a_{0}=0$ for $m=0$ ) one gets

$$
\lim _{\sigma \rightarrow 0} \boldsymbol{u}^{(m)}(r, \varphi, z, t, k, \sigma)=\mathbf{0} .
$$

The relation between the stationary pressure functions (10) and the corresponding instationary ones 
(Eq. (16) of [1] for $c_{c}=\infty$ ) is found to be

$$
\begin{aligned}
p^{(m, j)}(r, \varphi, z)= & p_{0}+\left.\frac{\partial\left[p_{(r, \varphi, z, t ; k, \sigma)}^{(m)} \mathrm{e}^{\sigma t}\right]}{\partial \sigma}\right|_{\substack{\sigma=0 \\
k=k^{(j)}}} \cdot B\left(m, k^{(j)}\right),
\end{aligned}
$$

where $B\left(m, k^{(j)}\right)$ are constants. Without explicit knowledge of the stationary functions $D_{\mathrm{S}}(k, m)$, $\boldsymbol{u}^{(m, j)}(r, \varphi, z), p^{(m, j)}(r, \varphi, z)$ it does not seem possible to state the relations (11), (12), (14). In order to get the relations (12) and (14), the process $\sigma \rightarrow 0$ with $b_{m} \sigma=b_{m}^{*}=$ const must be used, where the constants $b_{m}$ enter the functions $\boldsymbol{u}^{(m)}, p^{(m)}$ according to equation (18) of [1] for $c_{c}=\infty$. If one interprets the functions $\boldsymbol{u}^{(m)}$ or $\boldsymbol{u}^{(m, j)}$ for $k \neq 0$ as flow patterns in the pipe, the corresponding Reynolds-numbers must be assumed to be very small, which means that the coefficients $a_{0}, b_{m}$ must be sufficiently small.

The case of vanishing separation constant $k$ is of certain interest. If one solves (1) to (3) for $k=0$ and $\boldsymbol{u}(r=R, \varphi, z)=\mathbf{0}$, only the trivial solution $\boldsymbol{u} \equiv \mathbf{0}$, $p=$ const. results: especially the Hagen-Poiseuillesolution for $m=0$ does not appear. The reason is that the Hagen-Poiseuille-solution $\boldsymbol{u}_{\mathrm{HP}}(r)=$ $u_{0}\left(1-r^{2} / R^{2}\right) \boldsymbol{e}_{z}$ cannot be represented by (3). For $k=0, m=0$, however, (1) is solved by

$$
\begin{aligned}
\boldsymbol{u}^{(0,0)} & =\operatorname{rot} \cdot \operatorname{rot} \boldsymbol{b}_{r}^{(0,0)}(r), \quad p^{(0,0)}=p_{0}-c \varrho_{0} v_{0} z, \\
\boldsymbol{b}_{r}^{(0,0)} & =\boldsymbol{e}_{z} b_{r}^{(0,0)}(r), \quad \Delta_{r} \Delta_{r} b_{r}^{(0,0)}=c, \\
\Delta_{r} & =\frac{1}{r}\left(r \frac{\mathrm{d}}{\mathrm{d} r}\right),
\end{aligned}
$$

where $c$ is an arbitrary constant. The solution of $\Delta_{r} \Delta_{r} b_{r}^{(0,0)}(r)=c$ consists of the general solution of the homogeneous equation and a special solution of the inhomogeneous equation. Taking into account the non-trivial, non-singular terms only, we have

$$
\begin{aligned}
b_{r}^{(0,0)}(r)= & b_{0} r^{2}+\frac{c}{64} r^{4}+b_{1} r^{2} \ln r, \\
\Delta b_{r}^{(0,0)}= & \frac{c r^{2}}{4}+4 b_{0} \\
& +4 b_{1}(\ln r+1), \quad b_{1}=0,
\end{aligned}
$$

which gives

$\boldsymbol{u}_{(z)}^{(0,0)}=\frac{c}{4 R^{2}}\left(1-\frac{r^{2}}{R^{2}}\right) \boldsymbol{e}_{z}, \quad p_{(z)}^{(0,0)}=p_{0}-c \varrho_{0} v_{0} z$

As already Sexl [3] observed, the Hagen-Poiseuille solution can be found also from the time-dependent solutions of the Stokes equation for $m=0, k=0$ by the process $\lim _{\sigma \rightarrow 0} \boldsymbol{u}_{\mathrm{S}}^{(0)}(r, t, \sigma)$, where $\boldsymbol{u}_{\mathrm{S}}^{(0)}$ denotes the $c \sigma=$ const

functions found by Sexl ([3], p. 575). In order to find all stationary solutions for $k=0$ from the timedependent solutions of the Stokes equation by using the HSMF-method we take the solutions found by Brosa [1]. Since he did not separate the Helmholtzequation for $k=0$ quite correctly, we summarize the three types of time-dependent solutions for $k=0$ :

a) $\quad m=0, \alpha_{0}$ arbitrary, $b_{0}$ arbitrary:

$$
\begin{gathered}
J_{1}\left(\sqrt{\frac{\sigma_{1}^{(j)}}{v_{0}}} R\right)=0, \quad j=1,2,3, \ldots, \quad u_{r}^{(0)}=0, \\
u_{\varphi}^{(0)}=a_{0} \sqrt{\frac{\sigma_{1}^{(j)}}{v_{0}}} J_{1}\left(\sqrt{\frac{\sigma_{1}^{(j)}}{v_{0}}} r\right) \mathrm{e}^{-\sigma_{1}^{(j)} t}, \\
u_{z}^{(0)}=b_{0} \frac{\sigma_{1}^{(j)}}{v_{0}}\left[J _ { 0 } \left(\sqrt{\left.\frac{\sigma_{1}^{(j)}}{v_{0}} r\right)}\right.\right. \\
\left.-J_{0}\left(\sqrt{\frac{\sigma_{1}^{(j)}}{v_{0}}} R\right)\right] \mathrm{e}^{-\sigma_{1}^{(j)} t} .
\end{gathered}
$$

b) $\quad m=0, b_{0}$ arbitrary, $\sigma$ arbitrary:

$$
\begin{aligned}
u_{r}^{(0)}= & 0, \quad u_{\varphi}^{(0)}=0, \\
u_{z}^{(0)}= & b_{0} \frac{\sigma}{v_{0}}\left[J_{0}\left(\sqrt{\frac{\sigma}{v_{0}}} r\right)\right. \\
& \left.-J_{0}\left(\sqrt{\frac{\sigma}{v_{0}}} R\right)\right] \mathrm{e}^{-\sigma t} .
\end{aligned}
$$

c) $\quad m=0,1,2, \ldots, b_{m}$ arbitrary:

$$
\begin{aligned}
& J_{m}\left(\sqrt{\frac{\sigma_{m}^{(j)}}{v_{0}}} R\right)=0, \quad u_{r}^{(m)}=0, \quad u_{\varphi}^{(m)}=0, \\
& u_{z}^{(m)}=b_{m} \frac{\sigma_{m}^{(j)}}{v_{0}} J_{m}\left(\sqrt{\frac{\sigma_{m}^{(j)}}{v_{0}}} r\right) \mathrm{e}^{i m \varphi} \mathrm{e}^{-\sigma_{m}^{(j)} t} .
\end{aligned}
$$

If we look for stationary solutions with $k=0$ as result of a process $\sigma \rightarrow 0, b_{m} \sigma^{n}=$ const, only solutions of type b) can be used since in the other two cases $\sigma=0$ cannot be approached smoothly. Making explicit the argument used in [1] we get for

$$
\begin{aligned}
& u_{z}^{(0)}(r, t, \sigma) \\
& =b_{0} \frac{\sigma}{v_{0}}\left[J_{0}\left(\sqrt{\frac{\sigma}{v_{0}}} r\right)-J_{0}\left(\sqrt{\frac{\sigma}{v_{0}}} R\right)\right] \mathrm{e}^{-\sigma t}
\end{aligned}
$$


the relations

$$
\begin{aligned}
& u_{z}^{(0)}(r, t, \sigma=0)=0, \quad\left(\frac{\partial u_{z}^{(0)}(\sigma)}{\partial \sigma}\right)_{\sigma=0}=0, \\
& \left(\frac{\partial^{2} u_{z}^{(0)}(\sigma)}{\partial \sigma^{2}}\right)_{\sigma=0}=\frac{b_{0}}{2 v_{0}^{2}}\left(R^{2}-r^{2}\right) .
\end{aligned}
$$

Thus we find

$$
\begin{aligned}
& \lim _{\substack{\sigma \rightarrow 0 \\
b_{0} \sigma^{2}=b_{0}^{*}}} \boldsymbol{u}^{(0)}(r, t, \sigma)=\frac{b_{0}^{*}}{4 v_{0}^{2}}\left(R^{2}-r^{2}\right) \boldsymbol{e}_{z} \\
& \quad=u_{0}\left(1-\frac{r^{2}}{R^{2}}\right) \boldsymbol{e}_{z}=: \boldsymbol{u}_{\mathrm{HP}}(r), \\
& u_{0}=b_{0}^{*} R^{2} / 4 v_{0}^{2} .
\end{aligned}
$$

In the same way the stationary pressure $p_{\mathrm{HP}}(z)$ follows from

$$
p_{\mathrm{HP}}(z)=\lim _{\substack{\sigma \rightarrow 0 \\ b_{0} \sigma^{2}=b_{0}^{*}}} p^{(0)}(z, t, \sigma),
$$

where $p^{(0)}(z, t, \sigma)$ is given by ([1], Eq. (27))

$p^{(0)}(z, t, \sigma)=p_{0}-\varrho_{0} b_{0} \frac{\sigma^{2}}{v_{0}} J_{0}\left(\sqrt{\frac{\sigma}{v_{0}}} R\right) z \mathrm{e}^{-\sigma t}$.

With

$$
\begin{gathered}
p^{(0)}(z, t, \sigma=0)=p_{0}, \quad\left(\frac{\partial p^{(0)}(\sigma)}{\partial \sigma}\right)_{\sigma=0}=0, \\
\left(\frac{\partial^{2} p^{(0)}(\sigma)}{\partial \sigma^{2}}\right)_{\sigma=0}=-\frac{2 \varrho_{0} b_{0}}{v_{0}} z
\end{gathered}
$$

[1] U. Brosa, Z. Naturforsch. 41 a, 1141 (1986).

[2] J. A. Stratton, Electromagnetic Theory, McGraw-Hill New York 1941. P. M. Morse and H. Feshbach, we get finally

$$
p_{\mathrm{HP}}(z)=p_{0}-\frac{\varrho_{0}}{v_{0}} b_{0}^{*} z=p_{0}-4 \varrho_{0} v_{0} \frac{u_{0}}{R^{2}} z .
$$

Contrary to the stationary solutions $\boldsymbol{u}^{(m, j)}(r, \varphi, z)$, the coefficient $b_{0}^{*}$ (or $u_{0}$ ) which appears in $\boldsymbol{u}_{\mathrm{HP}}(r)$ may take values which give large Reynolds-numbers since $\boldsymbol{u}_{\mathrm{HP}}(r)$ solves the stationary non-linear NavierStokes equation.

With regard to the infinite pipe the HagenPoiseuille-solution remains as the only stationary solution of the Stokes equation which fulfills the (incomplete) boundary condition $\boldsymbol{u}(r=R)=\mathbf{0}$, which possesses continuous partial derivatives everywhere and which is bounded for $-\infty<z<\infty$. The functions $\boldsymbol{u}^{(m, j)}(r, \varphi, z)$ with $k \neq 0$ represent solutions of the Stokes equation for the semi-infinite pipe with $\boldsymbol{u}^{(m, j)}(r, \varphi, z \rightarrow \infty)=\mathbf{0}$ and a prescribed boundary condition at $z=0$.

We want to mention that also $\Delta a=0$ (2) is only sufficient to solve (1). If for the case $m=0, k=0$ one lets $\Delta a=c$, one finds an additional solution $\boldsymbol{u}(r)=-\frac{1}{2} \operatorname{cr} \boldsymbol{e}_{\varphi}$, which gives no contribution for homogeneous boundary conditions $\boldsymbol{u}^{(0,0)}(r)=\mathbf{0}$, but for a boundary condition

$$
\begin{aligned}
& u_{r}^{(0,0)}(r=R)=u_{z}^{(0,0)}(r=R)=0, \\
& u_{\varphi}^{(0,0)}(r=R)=u_{\varphi 0},
\end{aligned}
$$

which characterizes a rotating pipe.

Methods of Theoretical Physics, Part II, McGraw-Hill, New York 1953.

[3] Th. Sexl, Ann. Phys. 87, 570 (1928). 